\title{
Simulating and visualizing infection spread dynamics with temporal networks
}

\author{
Jean R. Ponciano ${ }^{1,2}$, Gabriel P. Vezono ${ }^{1}$, Claudio D. G. Linhares ${ }^{3}$ \\ ${ }^{1}$ Faculty of Computing, Federal University of Uberlândia, Uberlândia, Brazil \\ ${ }^{2}$ School of Applied Mathematics, Getulio Vargas Foundation (FGV), Brazil \\ ${ }^{3}$ Institute of Mathematics and Computer Sciences, University of São Paulo, \\ São Carlos, Brazil \\ jean.poncianodfgv.br, gvezono@gmail.com, claudiodgleusp.br
}

\begin{abstract}
Temporal networks comprehend a widely adopted structure to model interactions involving a domain's instances over time. In the context of infection spread, it could be used to model face-to-face contacts among susceptible and infected individuals. By considering network visualization strategies, one can easily identify who infected whom and when, the epidemics outbreak, and other relevant behaviors. As a consequence, decision making related to the spread speed and magnitude becomes faster and more reliable. This paper presents a visual analytics approach for the simulation and analysis of infection spread dynamics that considers different infection probabilities and different levels of social distancing. We performed our experiments using two real-world social networks that represent school environments and our findings support the need for a high social distancing compliance allied to the adoption of protective measures such as the use of face masks.
\end{abstract}

\section{Introduction}

Information visualization strategies create graphical and interactive representations of abstract data, thus comprising an effective resource to gain insights and guide decision making [Ponciano et al. 2020]. It is useful, for example, to provide awareness by graphically showing real-world problems. In the context of health/epidemic data, an efficient visual analysis may assist experts in the study and decisions related to infection spread dynamics, such as in the recent COVID-19 pandemic [Dong et al. 2020, So et al. 2020].

In epidemics, the infection propagates through an interaction (usually face-toface) involving an infected and a susceptible individual. Networks represent a useful and commonly used structure to model this type of data, i.e., data composed of interactions involving a domain's instances. In a network, the instances (e.g., individuals) are represented by nodes and the interactions that connect two nodes (e.g., face-to-face contacts) are represented by edges. When the information of when each interaction occurs is known, such network is called temporal network.

The temporal network dynamism, in which there are time intervals with bursts of interactions as well as periods of low (or none) activity, makes such network useful to simulate and study from fake news [Jr. and Frizzon 2019] to infection spreading [Linhares et al. 2019a]. Another relevant property of real-world networks is the 
community formation, i.e., the presence of groups of individuals that interact more often among themselves than with individuals from other groups [Linhares et al. 2019b]. Knowing these communities, that may be related to, e.g., household members or people in workplaces and school classes, allows a better understanding - and consequently a more effective decision making - regarding the control of the spread speed and magnitude [Ghalmane et al. 2019].

Temporal networks have been widely used to simulate infection spread dynamics through a variety of infection models [Linhares et al. 2019a]. Although the employment of visualization strategies comprehends a useful tool that allows, e.g., contact tracing and the understanding of the transmission path and spread speed [Carroll et al. 2014, Linhares et al. 2019a], few studies consider visualization strategies in their analysis. Most of them focus on stochastic models, geographical information, or statistical properties of the infected population [Tepper and Thiébaut 2017].

This paper presents a visual analytics approach for different simulations of infection spread dynamics that take place in real-world environments, represented in our study by temporal social networks. We simulate the infection propagation by using the susceptible-infected (SI) infection model [Prakash et al. 2014] and we consider different levels of social distancing and different infection probabilities, aspects that are perceived in real-world scenarios when the individuals avoid (or not) contacts or adopt (or not) protective measures such as the use of face masks. To the best of our knowledge, our work is the first to simultaneously consider both aspects in this type of study.

Our main contributions are: (i) a strategy for simulating different levels of social distancing that considers community detection and the number of inter-community interactions in a temporal network; (ii) a visual analytics approach that employs two visualization layouts (MSV and TAM [Linhares et al. 2020b]) along with a well-established node ordering method (CNO [Linhares et al. 2019b]) for the analysis of infection spread dynamics; (iii) an extended version of DyNetVis [Linhares et al. 2020b] that implements our methodology and is freely available for potential users. With our approach, one can easily perform different infection spread simulations and identify key nodes, the transmission path (who infected whom and when), the epidemics outbreak, and other relevant behaviors.

The rest of this paper is organized as follows. Section 2 presents related concepts and Section 3 discusses related work. Section 4 presents the strategies used for simulating and analyzing infection spread dynamics in the case studies. Section 5 describes the two evaluated real-world temporal networks and the performed experiments. Section 6 discusses the results and limitations. Section 7 presents the conclusion and future work.

\section{Related Concepts}

\subsection{Susceptible-Infected (SI) infection dynamics}

The susceptible-infected (SI) model comprehends a simple infection dynamics where each node is associated with one of two states at a particular time, susceptible (S) or infected (I). All nodes start susceptible, except for a node chosen to be initially infected (patient zero). At each subsequent timestamp, each interaction involving an infected node and one of its susceptible neighbors (adjacent nodes) has a probability $\lambda$ of infecting such neighbor. 
In the SI model, an infected node does not recovery or turn susceptible again. There are models that consider other situations, as, for example, the SIS model (an infected node turn susceptible again) and the SIR model (an infected node (R)ecovers after being infected for $t_{r}$ time steps) [Linhares et al. 2019a].

\subsection{Network Visualization}

Several strategies have been developed over the years to enhance the visualization of temporal networks and thus improve the user perception regarding network evolution and existing patterns [Beck et al. 2016]. Figure 1 presents different layouts and also illustrates their usefulness for analyzing infection spread dynamics.

The Massive Sequence View (MSV) [Linhares et al. 2019b] is a layout focused on the network temporal evolution. As shown in Figure 1(a), nodes are vertically displayed and timestamps are horizontally positioned. Whenever there is an edge connecting two nodes, a vertical line involving them is drawn at the corresponding timestamp. Figure 1(b) illustrates how MSV can be employed for analyzing the SI infection dynamics. In this case, MSV shows only those edges through which the infection propagates, thus highlighting the infection path from infected to susceptible nodes. This approach, along with a transparency effect on non-infected nodes, facilitates the identification of who infected whom and when the transmission occurred. Such information is useful to recognize the importance of particular nodes and act to regulate the infection spread.

The Temporal Activity Map (TAM) [Linhares et al. 2020b] is a variant of MSV where nodes are represented by squares instead of circles (thus giving a better sense of continuity) and all edges are omitted. When studying infection spread dynamics, TAM is useful to highlight the node state (Susceptible or Infected), facilitating the identification of how groups of nodes with the same state evolve (Figure 1(c)).

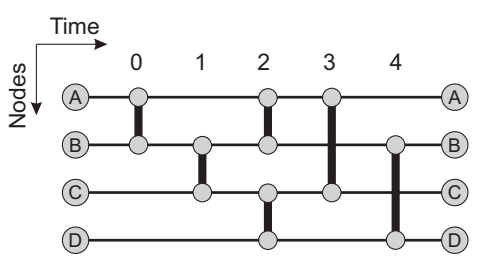

(a)

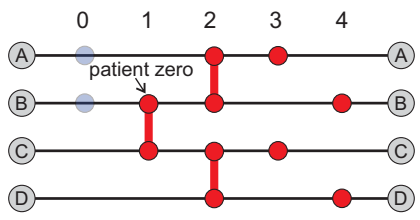

(b)

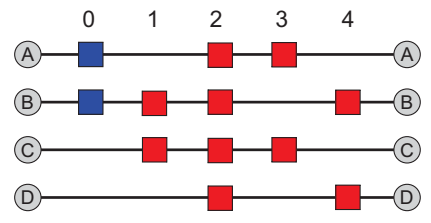

(c)

Susceptible

Infected $=$ Infection path

Figure 1. Different layouts for temporal network visualization: (a) MSV; (b) MSV with infection path; (c) TAM.

There are several efforts to reduce visual clutter and improve aesthetic aspects of network visualizations, such as the choice of nodes/edges positioning, sizes, colors, and others [Beck et al. 2016]. For MSV, there are strategies that focus on re-positioning nodes [Linhares et al. 2019b], sampling relevant edges [Ponciano et al. 2021b], and changing the network temporal resolution [Ponciano et al. 2021a]. The adoption of a high-quality node positioning strategy is important since changes in the node ordering can reduce edge length and consequently overlaps and visual clutter.

The community-based node ordering (CNO) [Linhares et al. 2019b] is a visual scalable node positioning strategy developed to optimize the analysis of temporal networks in MSV and TAM layouts. CNO detects network communities and then places their nodes in the layout in a way that nodes that belong to the same community are posi- 
tioned close to each other. Through interactive tools the user can associate different colors to different communities and also discard inter- or intra-community edges, thus reducing the amount of visual information and focusing the analysis on regions of interest.

\section{Related Work}

Different visualization tools have been developed over the past years to analyze health/epidemic data, as for example the interactive dashboard proposed in [Dong et al. 2020] to track COVID-19 reported cases. There are three main categories of visualization applications when dealing with health data [Carroll et al. 2014]: geographic information systems (GIS), that allow the identification of particular cases, spatial trends, and disease clusters, among others; molecular epidemiology, that considers, e.g., the visualization of serotypes and genetic variants; and social temporal network analysis, commonly used to understand infection outbreaks and trace contacts and infection paths.

VoroGraph [Dunne et al. 2015] is a visual analytics tool that considers different visualization strategies based on networks and geographic information to assist epidemiologists in their analysis. Another example of temporal network visualization strategy useful in this context is the radial layout developed in [Tepper and Thiébaut 2017], through which the user can identify individuals involved in the infection spread, trace contacts, and analyze how an intervention would influence the evaluated scenarios. More recently, temporal network visualization has also been used in the study of COVID-19 transmission risk in different countries (e.g., South Korea and China), often through spatial visualizations and/or node-link diagrams [Park 2020, So et al. 2020].

Without considering infection dynamics, MSV and TAM are commonly used to analyze general structural and temporal patterns in networks from different domains, including hospitals [Linhares et al. 2019b] and schools [Ponciano et al. 2020, Ponciano et al. 2021a]. Only recently these layouts have been considered for the simulation and analysis of infection spreads [Linhares et al. 2019a, Linhares et al. 2020b], but they were not applied in scenarios that consider different levels of social distancing. To the best of our knowledge, we are not aware of visualization studies that employ these layouts for analyzing infection propagation while considering both social distancing and different infection probabilities.

In [Chang et al. 2020], the authors consider social distancing as the limitation of non-household interactions while preserving household contacts unaltered. Even though household members comprehend a relevant group of nodes that should be considered in the analysis of infection spread, other groups based on sociological factors may exist (e.g., individuals in a workplace or in a school class). Groups such as these represent useful information and could be modeled as network communities [Kitchovitch and Liò 2010]. Although the analysis of the network community structure (e.g., the number and density of inter- and intra-community contacts) is crucial for defining effective immunization strategies, few studies consider this information [Ghalmane et al. 2019].

\section{Methodology}

To analyze how an infection spreads among nodes of a temporal network, we consider two key aspects. The first one concerns the number of interactions involving the existing nodes. Inspired by [Chang et al. 2020], we associate the number of interactions with 
social distancing, which is modeled in our context by keeping all CNO intra-community interactions unaltered while varying the allowed number of inter-community ones. To perform such variation, $\beta$ randomly chosen inter-community edges are maintained in the network, while the others are discarded. As an example, if $\beta=0$, all inter-community edges are discarded and consequently there is a full lockdown - which is impracticable in real-world scenarios since there are many essential services [Canabarro et al. 2020]. The second aspect is related to the probability $\lambda$ of a susceptible node be infected after interacting with an infected one. As an example, if $\lambda=1$, every susceptible node will certainly become infected after interacting with an infected node.

After running $\mathrm{CNO}$ over a given network and filtering in $\beta$ inter-community edges to simulate social distancing, one must select the patient zero (node $p$ ). Such a choice could be either arbitrary or related to a node structural property such as degree, or betweenness. In the sequence, a SI infection spread simulation considering $p$ and the chosen probability $\lambda$ can be performed. Finally, the user analyses the produced TAM or MSV layout to study the propagation dynamics, e.g., by tracking intra-community infection spread, identifying who infected whom and when, and perceiving other relevant behaviors (recall Figures 1 (b-c)). Given $p$, we repeat the entire process ten times for each combination $(\beta, \lambda)$ in order to have a more precise quantitative evaluation.

\section{Case studies}

This section presents two case studies using the real-world networks Primary School [Gemmetto et al. 2014] and High School [Mastrandrea et al. 2015]. We consider these networks because they were proposed to analyze infection spreads. Moreover, they are publicly available and also have been extensively explored in visualization studies (e.g., [Linhares et al. 2020a]). First, Section 5.1 describes the adopted parameter values and Section 5.2 presents the networks. In the sequence, Sections 5.3 and 5.4 present the experiments and results for both networks. All visual analyses were performed using DyNetVis [Linhares et al. 2020b], an interactive, freely available, and open-source software that was extended for this study to consider social distancing simulation. Our extended version is available at www.dynetvis.com (v4.1). Figures 2, 3, 4, and 5 can be accessed in higher resolutions at https://tinyurl.com/44k797ca.

\subsection{Parameter definition}

Although our focus is not the analysis of the COVID-19 transmission, we rely on studies that consider its behavior to define which level of social distancing ( $\beta$ value) should be considered in our experiments. While [Canabarro et al. 2020] consider social distancing with $75 \%$ of compliance, other studies (e.g., [Chang et al. 2020, Daghriri and Ozmen 2020]) recommend a compliance of at least $80 \%$. We go in the same direction as these studies and also consider that the number of interactions should be reduced in $80 \%$. We thus employ $\beta=0.2$ (recall that $\beta$ refers to the number of maintained inter-community interactions) and, as a counterpoint, $\beta=0.8$ (compliance of $20 \%$ ).

The infection probabilities considered in our experiments are $\lambda=0.05$ and $\lambda=0.7$, values that intend to simulate different levels of protective measures during the interactions, e.g., the use (or not) of face masks. For each analyzed network, the patient zero (node $p$ ) is set as the most active node of the most active CNO community. The time of the first infection is the first timestamp $p$ appears in the network. 
Finally, CNO is configured as follows. The adopted community detection algorithm (CNO first step) is Infomap for the Primary School and Louvain for the High School, as the detected communities are greatly related to the school classes in these cases [Linhares et al. 2020a]. To define node positioning (CNO second and third steps), Recurrent Neighbors (RN) is used [Linhares et al. 2020b].

\subsection{School networks}

The Primary School network [Gemmetto et al. 2014] was collected in Lyon, France and refers to face-to-face interactions among 232 students and ten teachers ( 242 nodes). There are 55,046 edges distributed in two days. The chosen temporal resolution for this network is a 5-minute interval, in a total of 389 timestamps. In this resolution, each timestamp represents 5 minutes and considers all edges that occur during this interval. There are five grades and each grade is divided into two classes (A and B). Each of the ten classes has an assigned teacher.

The High School network [Mastrandrea et al. 2015] was collected in Marseille, France and comprehends face-to-face interactions among 327 students. There are 47,586 edges distributed in five days and the adopted temporal resolution is also a 5-minute interval, totalizing 1,211 timestamps. There are nine classes in this network. They focus on different subjects and are divided into four categories: MP (mathematics and physics), PC (physics and chemistry), PSI (engineering studies), and BIO (biology).

The two networks were collected using RFID sensors from badges. Whenever two individuals have a frontal approximation (between 1 and 1.5 meters), an interaction (edge) is computed. Table 1 summarize both networks' properties. Note that the Primary School network presents a higher number of edges per timestamp on average (EPT) when compared to the High School. This behavior indicates that students from the primary school interact a lot more among themselves when compared with the students from the high school, a situation that could facilitate the infection propagation.

Table 1. School networks properties. 'EPT' refers to the average number of edges per timestamp. 'TR' refers to the adopted temporal resolution (in minutes).

\begin{tabular}{|l|l|l|l|l|l|l|}
\hline Network & \#Nodes & \#Edges & \#Times & EPT & \#Classes & TR \\
\hline Primary Sch. & 242 & 55,046 & 389 & 141.5 & 10 & 5 \\
\hline High Sch. & 327 & 47,586 & 1,211 & 39.29 & 9 & 5 \\
\hline
\end{tabular}

\subsection{Primary School case}

Table 2 presents the average result of a quantitative evaluation that considers ten simulations for each combination $(\beta, \lambda)$ in the Primary School network. By considering $\beta=0.8$ (social distancing of 20\%) and varying the infection probability $\lambda$, one can perceive, by looking at the median time of infection (MTI), that the spread speed has been slowed in more than two times when adopting $\lambda=0.05$. For $\beta=0.2$ (social distancing of $80 \%$ ) and $\lambda=0.05$, the transmission was more than four times slower when compared with $\lambda=0.7$, regardless of the adopted $\beta$. The combination that resulted in a lower spread speed and a higher number of non-infected nodes at the end of the observation period (10) was $(\beta=0.2, \lambda=0.05)$, showing the importance of having a high social distancing compliance along with protective measures that reduce infection probability.

Figure 2 shows four TAM layouts, one for each parameter combination. For $\lambda=0.7$, varying $\beta$ did not produced a significant difference in the infection spread be- 
Table 2. Quantitative evaluation for the Primary School network considering the average result of ten executions for each combination $(\beta, \lambda)$. 'ATI' and 'MTI' refer to the average and the median timestamp of infection, respectively.

\begin{tabular}{|c|c|c|c|c|c|}
\hline & $(\beta-\lambda)$ & ATI & MTI & \#inf. & \#non-inf. \\
\hline \multirow{4}{*}{ 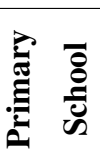 } & $(0.8-0.7)$ & $38.4 \pm 47.0$ & 24 & 241 & 1 \\
\hline & $(0.8-0.05)$ & $81.1 \pm 73.6$ & 60 & 240 & 2 \\
\hline & $(0.2-0.7)$ & $39.6 \pm 47.3$ & 25 & 241 & 1 \\
\hline & $(0.2-0.05)$ & $162.8 \pm 118.5$ & 109 & 232 & 10 \\
\hline
\end{tabular}

havior (see Figure 2 - first row), which is in agreement with the results from Tab. 2, In contrast, when assuming $\lambda=0.05$, different $\beta$ values produced different infection behaviors (Figure 2- second row). When considering $\beta=0.2$ and $\lambda=0.05$, for instance, several students entered in the second day of school being susceptible to the infection, most of them belonging to two particular classes (see blue nodes in the second day of the network for $(\beta=0.2, \lambda=0.05))$.
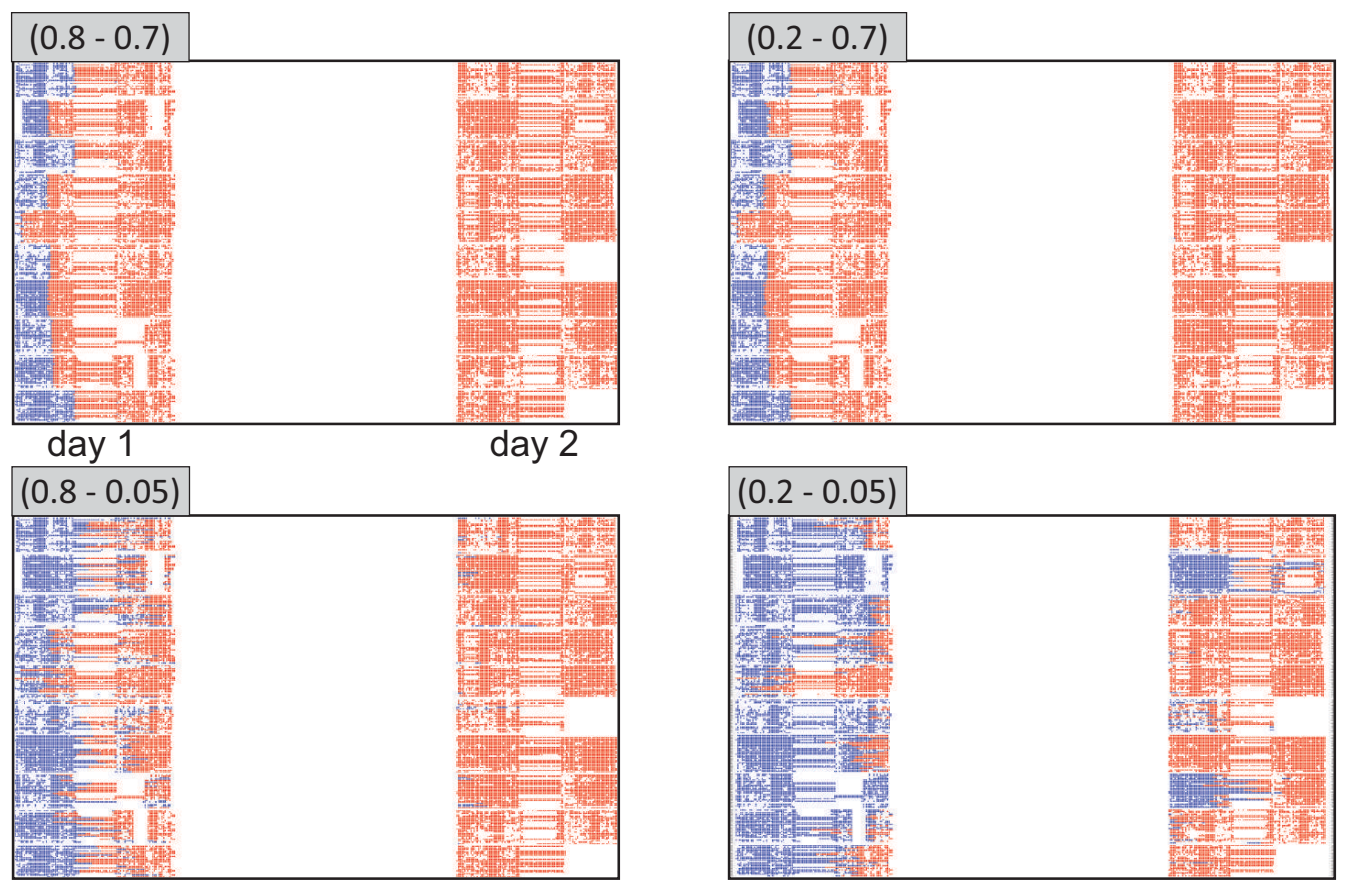

Figure 2. TAM layouts showing how different combinations $(\beta, \lambda)$ affect the infection spread behavior in the Primary School over two days.

Figure 3 illustrates the infection path for the combination $(\beta=0.2, \lambda=0.05)$ using the MSV layout. With this representation, one can easily identify the patient zero (central node of class 3B) and see the evolution of the infection spread in relation to the classes. As the patient zero is from class $3 \mathrm{~B}$, and considering that students from the same class interact more often among themselves than with others [Gemmetto et al. 2014], the infection propagates rapidly inside class $3 \mathrm{~B}$. A few moments later, a student from class $3 \mathrm{~A}$ becomes infected. Having both $3 \mathrm{~A}$ and $3 \mathrm{~B}$ infected one after another is not surprising, since same grade students also interact more often among themselves than with others [Gemmetto et al. 2014]. As illustrated in Figure 3, most of the inter-community interactions that allowed the infection to reach other classes happened during the first day's lunch break. During this interval, students from classes 1A, 1B, 4A, and 5B were infected. After the first day of school, only the students from classes $2 \mathrm{~A}$ and $2 \mathrm{~B}$ remained 
susceptible. These students were infected near the second day's lunch break.

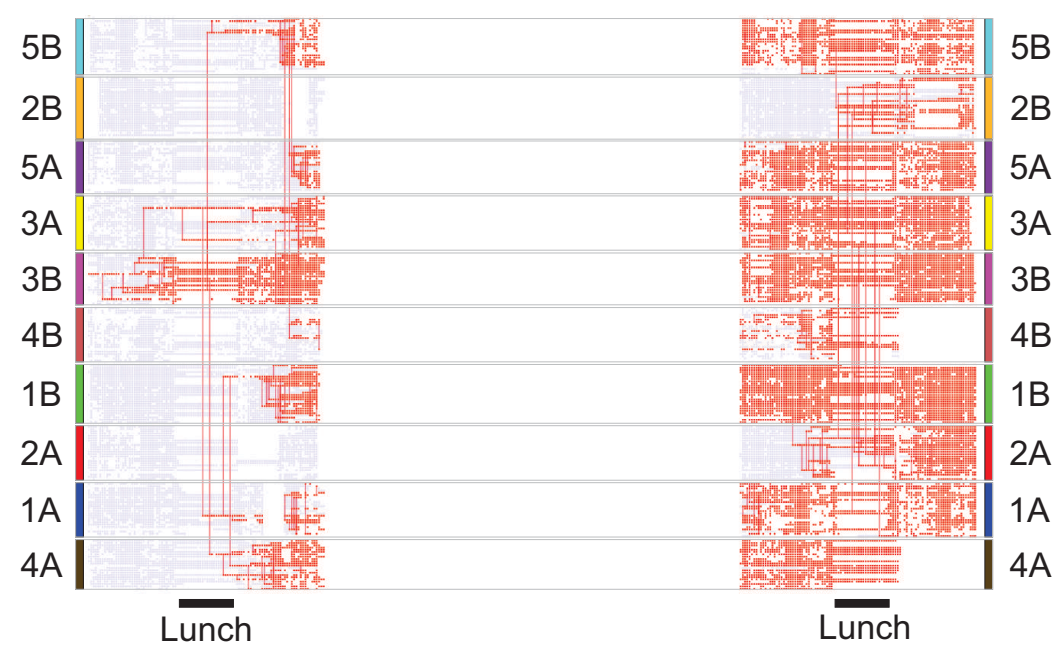

Figure 3. MSV infection path for the Primary School network $(\beta=0.2, \lambda=0.05)$.

\subsection{High School case}

Table 3 presents the average result of a quantitative evaluation that considers ten simulations for each combination $(\beta, \lambda)$ in the High School network. In the two cases where $\lambda=0.7$, all nodes become infected regardless of $\beta$. This is expected, since higher infection probabilities tend to require few interactions between two individuals to transmit the infection (recall that if $\lambda=1$, a single contact would infect a susceptible node, for instance). In these two cases, however, the spread speed varies according to $\beta$ in a way that the lower its value, the slower the infection spread (perceived by the higher median time of infection - MTI). By fixing $\beta$ and varying $\lambda$, one can see that a low $\lambda$ value leads to more non-infected nodes and slower spreads. As occurred with the Primary School, the combination $(\beta=0.2, \lambda=0.05)$ represents the best-case scenario: in this network, only $43.11 \%$ of the nodes (on average) became infected at the end of the observation period. This supports the importance of reducing the number of interactions while adopting protective measures to regulate the infection spread.

Table 3. Quantitative evaluation for the High School network considering the average result of ten executions for each combination $(\beta, \lambda)$. 'ATI' and 'MTI' refer to the average and the median timestamp of infection, respectively.

\begin{tabular}{|c|c|c|c|c|c|}
\hline & $(\beta-\lambda)$ & ATI & MTI & \#inf. & \#non-inf. \\
\hline \multirow{4}{*}{ 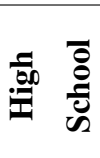 } & $(0.8-0.7)$ & $36.1 \pm 68.5$ & 21 & 327 & 0 \\
\hline & $(0.8-0.05)$ & $722.1 \pm 337.3$ & 773 & 260 & 67 \\
\hline & $(0.2-0.7)$ & $69.4 \pm 97.3$ & 29 & 327 & 0 \\
\hline & $(0.2-0.05)$ & $678 \pm 351.4$ & 689 & 141 & 186 \\
\hline
\end{tabular}

In the Primary School, the combination with MTI = 109 (of 389 timestamps) resulted in the slowest propagation of infected nodes, before one-third of the observation period. On the other hand, the slowest propagation in the High School infected the nodes with MTI $=773$ (of 1,211), therefore after half of the time. This difference in the spread speed can be justified by the different number of edges per timestamp (on average) that each network contains. Since the Primary School contains almost four times more edges than the High School (Tab. 1), its nodes tend to become infected earlier.

Figure 4 presents an overview of the four evaluated combinations for the High 
School. Through this visualization, one can easily see that almost all nodes become infected in the first day of the network when considering $\lambda=0.7$, regardless of the $\beta$ value (Figure 4-first row), but that a lower $\beta$ slows down the spread speed $(\beta=0.2, \lambda=0.7)$. In the same way, it is easy to note that the combination $(\beta=0.2, \lambda=0.05)$ produces the best-case scenario where less than half of the nodes become infected $(43.11 \%)$.
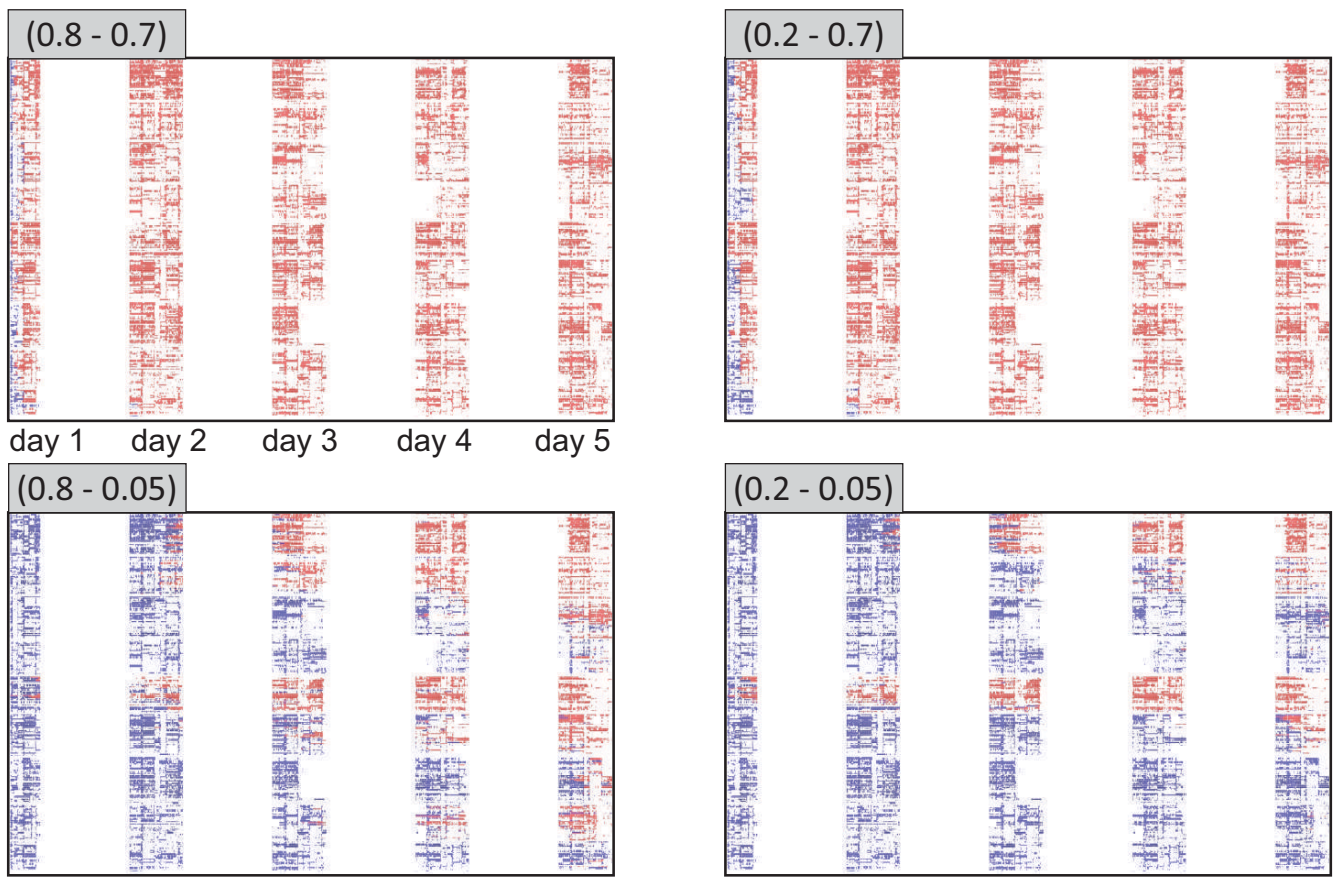

Figure 4. TAM layouts showing how different combinations $(\beta, \lambda)$ affect the infection spread behavior in the High School over five days.

Figure 5 shows the MSV layout for the combination $(\beta=0.2, \lambda=0.05)$ while highlights the transmission path and the involved classes. Through this visualization, one can see that the patient zero belongs to $2 \mathrm{BIO} 2$, a class of students that mainly study subjects related to biology [Mastrandrea et al. 2015]. Similar to the Primary School, where students interact more often with others from the same grade, in the High School students interact more often with others from classes focused on the same subjects [Mastrandrea et al. 2015]. This interaction behavior highly impacts the infection spread: the first three classes infected in the network are exactly the ones focused on biology (2BIO2 - that contains the patient zero -, 2BIO1, and 2BIO3). Note also that classes $\mathrm{PC}$ and $\mathrm{PC}^{*}$ (the ones based on physics and chemistry) had zero infected students.

\section{Discussion and Limitations}

We have presented case studies of infection propagation in two real-word temporal social networks. From the visualization perspective, the representations are very important to gain knowledge and generate insights over the data, with the possibility of finding unexpected patterns, trends, and anomalies that would be difficult to find otherwise. Through the analyzed visualizations, we were able to see the infection initially propagating across related classes, such as the ones from the same grade in Primary School or classes focused on the same subject in the High School. Moreover, the visualization allowed the identification of classes whose students were infected later than others as well as classes whose students were never infected. By understanding the infection path, domain ex- 


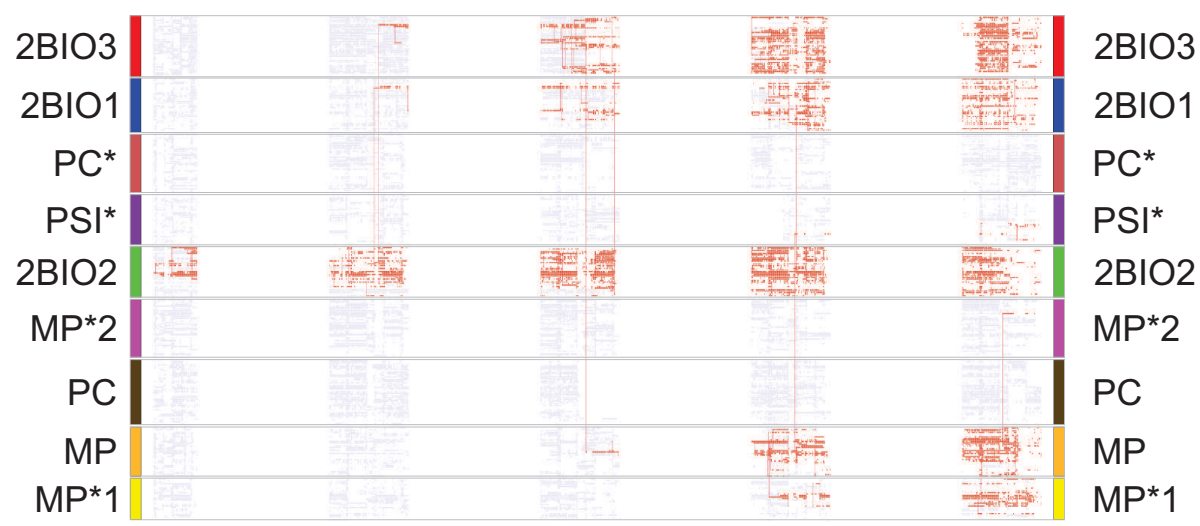

Figure 5. MSV infection path for the High School network $(\beta=0.2, \lambda=0.05)$.

perts can easily identify who infected whom and when, thus optimizing eventual decision making. In school environments, the school manager could act based on her/his findings. She/He could, e.g., change a class schedule to avoid interactions during lunch and, as a consequence, prevent new infections.

Our findings are inline with studies that show the importance of reducing both the infection probability and interactions involving different groups. In our case, we adopted $\lambda$ varying according to protective measures that could be used in real-world scenarios, such as the adoption of face masks, while $\beta$ stands for the social distancing compliance. Our findings, however, should only serve as a basis for future researches as several other aspects of infection propagation were not considered in our study. Examples include: (i) individuals or communities may adopt different precautions to reduce their susceptibility [Kitchovitch and Liò 2010]; (ii) different age-groups (or based on other criteria) may be differently affected [Canabarro et al. 2020]; (iii) the need for analyses considering other environments (e.g., networks from domains such as hospitals or shopping malls). As expected, the lower the number of interactions and infection probability, the less infected nodes at the end of the observation period. The main challenge is to find the parameter combination that best represents existing real-world scenarios and expected ones.

For both evaluated networks, the community detection methods returned communities highly related to the school classes. We have considered community detection instead of the direct use of class information because there are several real-world networks in which the community structure is not so clear. We thus aimed to demonstrate the suitability of our approach for the analysis of a variety of real-world networks, regardless of previous knowledge about existing groups of individuals. Nevertheless, the number of communities, their sizes, the quality of the detection, and the community structure strength may impact infection propagation and should be further analyzed [Ghalmane et al. 2019, Leão et al. 2019].

For convenience, our evaluation considered the Susceptible-Infected (SI) infection model, which is the most simple infection spread simulation model, to analyze the two presented real-world networks. There are more complex models that consider other variables, such as the death/recovery of an individual and latent/exposed states. When studying a particular disease, one must first identify which infection model is more suitable given the disease's characteristics. 


\section{Conclusion}

Network visualization strategies include the user in the network data exploration and validation through graphical and interactive representations. In the context of infection spread simulations, an effective visual analysis provides means for quick and reliable identification of key individuals, epidemics outbreak, transmission path, and contact tracing, among other relevant behaviors and patterns.

This paper presented a visual analytics approach for the simulation and analysis of infection spread dynamics. Our approach considers two key aspects: different levels of social distancing (i.e., whether the individuals avoid interacting with others), defined in our case by the number of inter-community interactions, and different infection probabilities (i.e., whether the individuals adopt protective measures such as the use of face masks). We demonstrated the usefulness of our approach through case studies that considered real-world interactions modeled by two temporal social networks. Our quantitative and visual analyses showed not only the importance of a high social distancing compliance allied to the adoption of protective measures, but also how local investigations lead to optimized understanding and reliable decision making.

In future work, we intend to analyze the impact of the community size in the infection spread. Furthermore, we also plan a follow-up study that considers other infection models (e.g., SIR and SIRS) and other layouts (e.g., animated node-link diagram) in simulations involving different environments. Finally, our plans also include automatically suggest potential initial patients for analysis and conduct a user evaluation to validate usefulness and usability.

\section{Acknowledgements}

This research was supported by Coordenação de Aperfeicoamento de Pessoal de Nível Superior, and São Paulo Research Foundation (FAPESP - Grants number 2020/10049-0, 2016/17078-0). The authors also thank SocioPatterns for making available the network data sets used in this paper.

\section{References}

[Beck et al. 2016] Beck, F., Burch, M., Diehl, S., and Weiskopf, D. (2016). A taxonomy and survey of dynamic graph visualization. Computer Graphics Forum, 36(1):133-159.

[Canabarro et al. 2020] Canabarro, A., Tenorio, E., Martins, R., Martins, L., Brito, S., and Chaves, R. (2020). Data-driven study of the covid-19 pandemic via age-structured modelling and prediction of the health system failure in brazil amid diverse intervention strategies. medRxiv.

[Carroll et al. 2014] Carroll, L. N., Au, A. P., Detwiler, L. T., Fu, T.-c., Painter, I. S., and Abernethy, N. F. (2014). Visualization and analytics tools for infectious disease epidemiology: a systematic review. Journal of biomedical informatics, 51:287-298.

[Chang et al. 2020] Chang, S., Harding, N., Zachreson, C., Cliff, O. M., and Prokopenko, M. (2020). Modelling transmission and control of the covid-19 pandemic in australia. ArXiv, abs/2003.10218.

[Daghriri and Ozmen 2020] Daghriri, T. and Ozmen, O. (2020). Quantifying the effects of social distancing on the spread of covid-19. Journal of Vaccines \& Vaccination.

[Dong et al. 2020] Dong, E., Du, H., and Gardner, L. (2020). An interactive web-based dashboard to track covid-19 in real time. The Lancet infectious diseases, 20(5):533-534. 
[Dunne et al. 2015] Dunne, C., Muller, M., Perra, N., and Martino, M. (2015). Vorograph: Visualization tools for epidemic analysis. In Proceedings of the 33rd Annual ACM Conference Extended Abstracts on Human Factors in Computing Systems, CHI EA '15, page 255-258, New York, NY, USA. Association for Computing Machinery.

[Gemmetto et al. 2014] Gemmetto, V., Barrat, A., and Cattuto, C. (2014). Mitigation of infectious disease at school: targeted class closure vs school closure. BMC infectious diseases, 14(1):695.

[Ghalmane et al. 2019] Ghalmane, Z., El Hassouni, M., and Cherifi, H. (2019). Immunization of networks with non-overlapping community structure. Social Network Analysis and Mining, 9(1):45.

[Jr. and Frizzon 2019] Jr., L. G. and Frizzon, G. (2019). Fake news and brazilian politics - temporal investigation based on semantic annotations and graph analysis. In Anais do XXXIV SBBD, pages 169-174, Porto Alegre, RS, Brasil. SBC.

[Kitchovitch and Liò 2010] Kitchovitch, S. and Liò, P. (2010). Risk perception and disease spread on social networks. Procedia Computer Science, 1(1):2345 - 2354. ICCS 2010.

[Leão et al. 2019] Leão, J., Laender, A., and de Melo, P. (2019). A multi-strategy approach to overcoming bias in community detection evaluation. In Anais do XXXIV SBBD, pages 13-24, Porto Alegre, RS, Brasil. SBC.

[Linhares et al. 2020a] Linhares, C., Ponciano, J., Pereira, F., Rocha, L., Paiva, J., and Travençolo, B. (2020a). Visual analysis for evaluation of community detection algorithms. MTAP, 79(25):17645-17667.

[Linhares et al. 2020b] Linhares, C. D. G., Ponciano, J. R., Paiva, J. G. S., Rocha, L. E. C., and Travençolo, B. A. N. (2020b). DyNetVis - an interactive software to visualize structure and epidemics on temporal networks. In 2020 IEEE/ACM ASONAM, pages 933-936.

[Linhares et al. 2019a] Linhares, C. D. G., Ponciano, J. R., Paiva, J. G. S., Travençolo, B. A. N., and Rocha, L. E. C. (2019a). Visualisation of Structure and Processes on Temporal Networks, pages 83-105. Springer International Publishing, Cham.

[Linhares et al. 2019b] Linhares, C. D. G., Ponciano, J. R., Pereira, F. S. F., Rocha, L. E. C., Paiva, J. G. S., and Travençolo, B. A. (2019b). A scalable node ordering strategy based on community structure for enhanced temporal network visualization. Computers \& Graphics, 84:185 - 198.

[Mastrandrea et al. 2015] Mastrandrea, R., Fournet, J., and Barrat, A. (2015). Contact patterns in a high school: A comparison between data collected using wearable sensors, contact diaries and friendship surveys. PLOS ONE, 10(9):1-26.

[Park 2020] Park, J. Y. (2020). Spatial visualization of cluster-specific covid-19 transmission network in south korea during the early epidemic phase. medRxiv.

[Ponciano et al. 2021a] Ponciano, J. R., Linhares, C. D., Faria, E. R., and Travençolo, B. A. (2021a). An online and nonuniform timeslicing method for network visualisation. $C \& G, 97: 170-182$.

[Ponciano et al. 2020] Ponciano, J. R., Linhares, C. D., Melo, S. L., Lima, L. V., and Travençolo, B. A. (2020). Visual analysis of contact patterns in school environments. Informatics in Education, 19(3):455-472.

[Ponciano et al. 2021b] Ponciano, J. R., Linhares, C. D. G., Rocha, L. E. C., Faria, E. R., and Travençolo, B. A. N. (2021b). A streaming edge sampling method for network visualization. KAIS, 63:1717-1743.

[Prakash et al. 2014] Prakash, B. A., Vreeken, J., and Faloutsos, C. (2014). Efficiently spotting the starting points of an epidemic in a large graph. Knowledge and information systems, 38(1):35-59.

[So et al. 2020] So, M., Tiwari, A., Chu, A., Tsang, J., and Chan, J. (2020). Visualizing covid-19 pandemic risk through network connectedness. International Journal of Infectious Diseases, 96:558 - 561.

[Tepper and Thiébaut 2017] Tepper, J. G. and Thiébaut, D. (2017). Data visualization of agent-based simulation of an infectious spread. In INFOCOMP 2017. 